\title{
Some Identities of the Degenerate Multi-Poly-Bernoulli Polynomials of Complex Variable
}

\author{
G. Muhiuddin $\left(\mathbb{D},{ }^{1}\right.$ W. A. Khan, ${ }^{2}$ U. Duran $\mathbb{D}^{,},{ }^{3}$ and D. Al-Kadi ${ }^{4}$ \\ ${ }^{1}$ Department of Mathematics, University of Tabuk, Tabuk 71491, Saudi Arabia \\ ${ }^{2}$ Department of Mathematics and Natural Sciences, Prince Mohammad Bin Fahd University, P.O Box 1664, \\ Al Khobar 31952, Saudi Arabia \\ ${ }^{3}$ Department of the Basic Concepts of Engineering, Faculty of Engineering and Natural Sciences, Iskenderun Technical University, \\ TR-31200 Hatay, Turkey \\ ${ }^{4}$ Department of Mathematics and Statistic, College of Science, Taif University, P.O. Box 11099, Taif 21944, Saudi Arabia
}

Correspondence should be addressed to G. Muhiuddin; chishtygm@gmail.com

Received 17 April 2021; Accepted 18 May 2021; Published 2 June 2021

Academic Editor: Gangadharan Murugusundaramoorthy

Copyright (c) 2021 G. Muhiuddin et al. This is an open access article distributed under the Creative Commons Attribution License, which permits unrestricted use, distribution, and reproduction in any medium, provided the original work is properly cited.

In this paper, we introduce degenerate multi-poly-Bernoulli polynomials and derive some identities of these polynomials. We give some relationship between degenerate multi-poly-Bernoulli polynomials degenerate Whitney numbers and Stirling numbers of the first kind. Moreover, we define degenerate multi-poly-Bernoulli polynomials of complex variables, and then, we derive several properties and relations.

\section{Introduction}

For any $\lambda \in \mathbb{R} /\{0\}$ (or $\mathbb{C} /\{0\}$ ), degenerate version of the exponential function $e_{\lambda}^{x}(t)$ is defined as follows (see [1-15])

$$
e_{\lambda}^{x}(t):=(1+\lambda t)^{\frac{x}{\lambda}}=\sum_{n=0}^{\infty}(x)_{n, \lambda} \frac{t^{n}}{n !},
$$

where $(x)_{0, \lambda}=1$ and $(x)_{n, \lambda}=x(x-\lambda) \cdots(x-(n-1) \lambda)$ for $n$ $\geq 1,\left(c f\right.$. [1-15]). It follows from (1) is $\lim _{\lambda \rightarrow 0} e_{\lambda}^{x}(t)=e^{\mathrm{xt}}$. Note that $e_{\lambda}^{1}(t):=e_{\lambda}(t)$.

Carlitz [1] introduced the degenerate Bernoulli polynomials as follows:

$$
\frac{t}{e_{\lambda}(t)-1} e_{\lambda}^{x}(t)=\sum_{n=0}^{\infty} \beta_{n}(x ; \lambda) \frac{t^{n}}{n !} .
$$

Upon setting $x=0, \beta_{n}(0 ; \lambda):=\beta_{n}(\lambda)$ are called the degenerate Bernoulli numbers.
Note that

$$
\lim _{\lambda \rightarrow 0} \beta_{n}(x ; \lambda)=B_{n}(x),
$$

where $B_{n}(x)$ are the familiar Bernoulli polynomials (cf. $[1,3$, $4,6,8,11,12,14,16-22])$

$$
\frac{t}{e^{t}-1} e^{x t}=\sum_{n=0}^{\infty} B_{n}(x) \frac{t^{n}}{n !}, \quad(|t|<2 \pi) .
$$

For $k \in \mathbb{Z}$, the polyexponential function $\operatorname{Ei}_{k}(x)$ is defined by (see [21])

$$
\mathrm{Ei}_{k}(x)=\sum_{n=1}^{\infty} \frac{x^{n}}{(n-1) ! n^{k}},(k \in \mathbb{Z})
$$

Setting $k=1$ in (5), we have $\operatorname{Ei}_{1}(x)=e^{x}-1$. 
The degenerate modified polyexponential function [12] is defined, for $k \in \mathbb{Z}$ and $|x|<1$, by

$$
\operatorname{Ei}_{k ; \lambda}(x)=\sum_{n=1}^{\infty} \frac{(1)_{n, \lambda}}{(n-1) ! n^{k}} x^{n}
$$

Note that $\operatorname{Ei}_{1 ; \lambda}(x)=e_{\lambda}(x)-1$.

Let $k \in \mathbb{Z}$ and $\lambda \in \mathbb{R}$. The degenerate poly-Bernoulli polynomials, $c f$. [12], are defined by

$$
\begin{gathered}
\frac{\mathrm{Ei}_{k ; \lambda}\left(\log _{\lambda}(1+t)\right)}{e_{\lambda}(t)-1} e_{\lambda}^{x}(t)=\sum_{n=0}^{\infty} B_{n, \lambda}^{(k)}(x) \frac{t^{n}}{n !}, \\
\log _{\lambda}(1+t)=\sum_{n=1}^{\infty} \lambda^{n-1}(1)_{n, \frac{1}{\lambda}} \frac{t^{n}}{n !},(\lambda \in \mathbb{R}),
\end{gathered}
$$

where $\log _{\lambda}(1+t)$ are called the degenerate version of the logarithm function ( $c f .[8,12])$, which is also the inverse function of the degenerate exponential function $e_{\lambda}(t)$ as shown below $(c f .[8])$

$$
e_{\lambda}\left(\log _{\lambda}(1+t)\right)=\log _{\lambda}\left(e_{\lambda}(1+t)\right)=1+t
$$

Letting $x=0$ in (7), $B_{n, \lambda}^{(k)}(0):=B_{n, \lambda}^{(k)}$ are called the type 2 degenerate poly-Bernoulli numbers.

The degenerate Stirling numbers of the first kind ( $c f$. $[8,13])$ and second kind ( $c f .[4-6,9,17])$ are defined, respectively, by

$$
\frac{1}{k !}\left(\log _{\lambda}(1+t)\right)^{\mathrm{k}}=\sum_{n=k}^{\infty} S_{1, \lambda}(n, k) \frac{t^{n}}{n !},(k \geq 0)
$$

and $(c f .[1-27])$

$$
\frac{1}{k !}\left(e_{\lambda}(t)-1\right)^{\mathrm{k}}=\sum_{n=k}^{\infty} S_{2, \lambda}(n, k) \frac{t^{n}}{n !},(k \geq 0) .
$$

Note that $\lim _{\lambda \rightarrow 0}$ in (10) and (1.8), we have (cf. $\left.[8,13]\right)$

$$
\frac{(\log (1+t))^{k}}{k !}=\sum_{n=k}^{\infty} S_{1}(n, k) \frac{t^{n}}{n !}(k \geq 0)
$$

and $(c f .[4-6,9,17,24])$

$$
\frac{\left(e^{t}-1\right)^{k}}{k !}=\sum_{n=k}^{\infty} S_{2}(n, k) \frac{t^{n}}{n !}(k \geq 0)
$$

where $S_{1}(n, k)$ and $S_{2}(n, k)$ are called the Stirling numbers of the first kind and second kind.

The following paper is as follows. In Section 2, we define the degenerate multi-poly-Bernoulli polynomials and numbers by using the degenerate multiple polyexponential functions and derive some properties and relations of these polynomials. In Section 3, we consider the degenerate multi-poly-Bernoulli polynomials of a complex variable and then we derive several properties and relations. Also, we examine the results derived in this study [28, 29].

\section{Degenerate Multi-Poly-Bernoulli Polynomials and Numbers}

Let $k_{1}, k_{2}, \cdots, k_{r} \in \mathbb{Z}$. The degenerate multiple polyexponential function $\mathrm{Ei}_{k_{1}, k_{2}, \cdots, k_{r} ; \lambda}(x)$ is defined (cf. [15]) by

$$
\mathrm{Ei}_{k_{1}, k_{2}, \cdots, k_{r} ; \lambda}(x)=\sum_{0<n_{1}<n_{2}<\cdots<n_{r}} \frac{(1)_{n_{1}, \lambda} \cdots(1)_{n_{r}, \lambda} x^{n_{r}}}{\left(n_{1}-1\right) ! \cdots\left(n_{r}-1\right) ! n_{1}^{k_{1}} \cdots n_{r}^{k_{r}}},
$$

where the sum is over all integers $n_{1}, n_{2}, \cdots, n_{r}$ satisfying $0<n_{1}<n_{2}<\cdots<n_{r}$. Utilizing this function, Kim et al. [15] introduced and studied the degenerate multi-poly-Genocchi polynomials given by

$$
\frac{2^{r} \mathrm{Ei}_{k_{1}, k_{2}, \cdots, k_{r} ; \lambda}\left(\log _{\lambda}(1+t)\right)}{\left(e_{\lambda}(t)+1\right)^{r}} e_{\lambda}^{x}(t)=\sum_{n=0}^{\infty} g_{n, \lambda}^{\left(k_{1}, k_{2}, \cdots k_{r}\right)}(x) \frac{t^{n}}{n !} .
$$

Inspired by the definition of degenerate multi-poly-Genocchi polynomials, using the degenerate multiple polyexponential function (14), we give the following definition.

Definition 1. Let $k_{1}, k_{2}, \cdots, k_{r} \in \mathbb{Z}$ and $\lambda \in \mathbb{R}$, we consider the degenerate multi-poly-Bernoulli polynomials are given by

$$
\frac{r ! \mathrm{Ei}_{k_{1}, k_{2}, \cdots, k_{r} ; \lambda}\left(\log _{\lambda}(1+t)\right)}{\left(e_{\lambda}(t)-1\right)^{r}} e_{\lambda}^{x}(t)=\sum_{n=0}^{\infty} \mathfrak{B}_{n, \lambda}^{\left(k_{1}, k_{2}, \cdots, k_{r}\right)}(x) \frac{t^{n}}{n !} .
$$

Upon setting $x=0$ in (16), the degenerate multi-polyBernoulli polynomials reduce to the corresponding numbers, namely, the type 2 degenerate multi-poly-Bernoulli numbers $\mathfrak{B}_{n, \lambda}^{\left(k_{1}, k_{2}, \cdots, k_{r}\right)}(0):=\mathfrak{B}_{n, \lambda}^{\left(k_{1}, k_{2}, \cdots, k_{r}\right)}$.

Remark 2. As $\lambda \rightarrow 0$, the degenerate multi-poly-Bernoulli polynomials reduce to the multi-poly-Bernoulli polynomials given by

$$
\frac{r ! \mathrm{Ei}_{k_{1}, k_{2}, \cdots, k_{r}}(\log (1+t))}{\left(e^{t}-1\right)^{r}} e^{x t}=\sum_{n=0}^{\infty} \mathfrak{B}_{n}^{\left(k_{1}, k_{2}, \cdots, k_{r}\right)}(x) \frac{t^{n}}{n !} .
$$

Remark 3. Upon setting $r=1$ in (16), the degenerate multipoly-Bernoulli polynomials reduce to the degenerate polyBernoulli polynomials in (7).

Before going to investigate the properties of the degenerate multi-poly-Bernoulli polynomials, we first give the following result. 
Proposition 4 (Derivative Property). For $k_{1}, k_{2}, \cdots, k_{r} \in \mathbb{Z}$ and $\lambda \in \mathbb{R}$, we have

$$
\frac{d}{d x} E i_{k_{1}, k_{2}, \cdots, k_{r} ; \lambda}(x)=\frac{1}{x} E i_{k_{1}, k_{2}, \cdots, k_{r}-1 ; \lambda}(x) .
$$

Proof. By (14), we see that

$$
\begin{aligned}
\frac{d}{d x} \operatorname{Ei}_{k_{1}, k_{2}, \cdots, k_{r} ;}(x)= & \frac{d}{d x} \sum_{0<n_{1}<n_{2}<\cdots<n_{r}} \\
& \cdot \frac{(1)_{n_{1}, \lambda} \cdots(1)_{n_{r}, \lambda} x^{n_{r}}}{\left(n_{1}-1\right) ! \cdots\left(n_{r}-1\right) ! n_{1}^{k_{1}} \cdots n_{r}^{k_{r}}} \\
= & \frac{1}{x_{0<n_{1}<n_{2}<\cdots<n_{r}}} \\
& \cdot \frac{(1)_{n_{1}, \lambda} \cdots(1)_{n_{r}, \lambda} x^{n_{r}}}{\left(n_{1}-1\right) ! \cdots\left(n_{r}-1\right) ! n_{1}^{k_{1}} \cdots n_{r}^{k_{r}-1}} \\
= & \frac{1}{x} \operatorname{Ei}_{k_{1}, k_{2}, \cdots, k_{r}-1 ; \lambda}(x) .
\end{aligned}
$$

Theorem 5. The following relationship

$$
\mathfrak{B}_{n, \lambda}^{\left(k_{1}, k_{2}, \cdots, k_{r}\right)}(x)=\sum_{j=0}^{n}\left(\begin{array}{c}
n \\
j
\end{array}\right) \mathfrak{B}_{n-j, \lambda}^{\left(k_{1}, k_{2}, \cdots k_{r}\right)}(x)_{j, \lambda}
$$

holds for $n \geq 0$.

Proof. Recall Definition 1 that

$$
\begin{aligned}
\sum_{n=0}^{\infty} \mathfrak{B}_{n, \lambda}^{\left(k_{1}, k_{2}, \cdots, k_{r}\right)}(x) \frac{t^{n}}{n !} & =\frac{r ! \mathrm{Ei}_{k_{1}, k_{2}, \cdots, k_{r}, \lambda}\left(\log _{\lambda}(1+t)\right)}{\left(e_{\lambda}(t)-1\right)^{r}} e_{\lambda}^{x}(t) \\
& =\sum_{n=0}^{\infty} \mathfrak{B}_{n, \lambda}^{\left(k_{1}, k_{2}, \cdots, k_{r}\right)} \frac{t^{n}}{n !} \sum_{j=0}^{\infty}(x)_{j, \lambda} \frac{t^{m}}{m !} \\
& =\sum_{n=0}^{\infty}\left(\sum_{j=0}^{n}\left(\begin{array}{c}
n \\
j
\end{array}\right) \mathfrak{B}_{n-j, \lambda}^{\left(k_{1}, k_{2}, \cdots k_{r}\right)}(x)_{j, \lambda}\right) \frac{t^{n}}{n !},
\end{aligned}
$$

which gives the asserted result (20).

The degenerate Bernoulli polynomials of order $r$ are given by the following series expansion:

$$
\sum_{n=0}^{\infty} \beta_{n}^{(r)}(x ; \lambda) \frac{t^{n}}{n !}=\left(\frac{t}{e_{\lambda}(t)-1}\right)^{r} e_{\lambda}^{x}(t)
$$

(cf. $[3,6,8,17])$.

We provide the following theorem.
Theorem 6. For $n \geq r$. Then

$$
\begin{aligned}
\mathfrak{B}_{n, \lambda}^{\left(k_{1}, k_{2}, \cdots, k_{r}\right)}(x)= & \sum_{m=0}^{n+r} \sum_{0<n_{1}<n_{2}<\cdots<n_{r} \leq m} \\
& \cdot\left(\begin{array}{c}
n+r \\
m
\end{array}\right) \beta_{n+r-m}^{(r)}(x ; \lambda) S_{1 . \lambda}\left(m, n_{r}\right) \\
& \times \frac{n ! r ! n_{r} !(1)_{n_{1}, \lambda} \cdots(1)_{n_{r}, \lambda}}{(n+r) !\left(n_{1}-1\right) ! \cdots\left(n_{r}-1\right) ! n_{1}^{k_{1}} \cdots n_{r}^{k_{r}}} .
\end{aligned}
$$

Proof. Recall from Definition 1 and (10) that

$$
\begin{aligned}
& \sum_{n=0}^{\infty} \mathfrak{B}_{n, \lambda}^{\left(k_{1}, k_{2}, \cdots, k_{r}\right)}(x) \frac{t^{n}}{n !}=\frac{r ! e_{\lambda}^{x}(t)}{\left(e_{\lambda}(t)-1\right)^{r}} \sum_{0<n_{1}<n_{2}<\cdots<n_{r}} \\
& \cdot \frac{(1)_{n_{1} \lambda} \cdots(1)_{n_{r} \lambda}\left(\log _{\lambda}(1+t)\right)^{n_{r}}}{\left(n_{1}-1\right) ! \cdots\left(n_{r}-1\right) ! n_{1}^{k_{1}} \cdots n_{r}^{k_{r}}} \\
& =\frac{r ! e_{\lambda}^{\chi}(t)}{\left(e_{\lambda}(t)-1\right)^{r}} \sum_{0<n_{1}<n_{2}<\cdots<n_{r}} \\
& \cdot \frac{(1)_{n_{1}, \lambda} \cdots(1)_{n_{r} \lambda} n_{r} !}{\left(n_{1}-1\right) ! \cdots\left(n_{r}-1\right) ! n_{1}^{k_{1}} \cdots n_{r}^{k_{r}}} \\
& \cdot \sum_{m=n_{r}}^{\infty} S_{1 . \lambda}\left(m, n_{r}\right) \frac{t^{m}}{m !} \\
& =\frac{r !}{t^{r}}\left(\frac{t^{r} e_{\lambda}^{\chi}(t)}{\left(e_{\lambda}(t)-1\right)^{r}}\right) \sum_{m=n_{r}}^{\infty} \\
& \cdot\left(\sum_{0<n_{1}<n_{2}<\cdots<n_{r} \leq m} \frac{(1)_{n_{1}, \lambda} \cdots(1)_{n_{r},} S_{1 . \lambda}\left(m, n_{r}\right) n_{r} !}{\left(n_{1}-1\right) ! \cdots\left(n_{r}-1\right) ! n_{1}^{k_{1}} \cdots n_{r}^{k_{r}}}\right) \\
& \cdot \frac{t^{m}}{m !}=\frac{r !}{t^{r}} \sum_{l=0}^{\infty} \beta_{l}^{(r)}(x ; \lambda) \frac{t^{l}}{l !} \sum_{m=n_{r}}^{\infty} \\
& \cdot\left(\sum_{0<n_{1}<n_{2}<\cdots<n_{r} \leq m} \frac{(1)_{n_{1}, \lambda} \cdots(1)_{n_{r},} S_{1 . \lambda}\left(m, n_{r}\right) n_{r} !}{\left(n_{1}-1\right) ! \cdots\left(n_{r}-1\right) ! n_{1}^{k_{1}} \cdots n_{r}^{k_{r}}}\right) \\
& \cdot \frac{t^{m}}{m !}=\sum_{n=r}^{\infty} \sum_{m=0}^{n}\left(\begin{array}{l}
n \\
m
\end{array}\right)_{0<n_{1}<n_{2}<\cdots<n_{r} \leq m} \\
& \cdot \frac{r ! n_{r} !(1)_{n_{1}, \lambda} \cdots(1)_{n_{r} \lambda}}{\left(n_{1}-1\right) ! \cdots\left(n_{r}-1\right) ! n_{1}^{k_{1}} \cdots n_{r}^{k_{r}}} \\
& \cdot \beta_{n-m}^{(r)}(x ; \lambda) S_{1 . \lambda}\left(m, n_{r}\right) \frac{t^{n-r}}{n !} \text {, }
\end{aligned}
$$

which means the claimed result (23).

Theorem 7. The following formula

$$
\mathfrak{B}_{n, \lambda}^{\left(k_{1}, k_{2}, \cdots, k_{r}\right)}(x+y)=\sum_{j=0}^{n}\left(\begin{array}{c}
n \\
j
\end{array}\right)(y)_{j, \lambda} \mathfrak{B}_{n-j, \lambda}^{\left(k_{1}, k_{2}, \cdots, k_{r}\right)}(x),
$$

is valid for $k_{1}, k_{2}, \cdots, k_{r} \in \mathbb{Z}$ and $n \geq 0$. 
Proof. In view of Definition 1, we see that

$$
\begin{aligned}
\sum_{n=0}^{\infty} \mathfrak{B}_{n, \lambda}^{\left(k_{1}, k_{2}, \cdots, k_{r}\right)}(x+y) \frac{t^{n}}{n !} & =\frac{r ! \operatorname{Ei}_{k_{1}, k_{2}, \cdots, k_{r}, \lambda}\left(\log _{\lambda}(1+t)\right)}{\left(e_{\lambda}(t)-1\right)^{r}} e_{\lambda}^{x+y}(t) \\
& =\sum_{i=0}^{\infty} \mathfrak{B}_{i, \lambda}^{\left(k_{1}, k_{2}, \cdots, k_{r}\right)}(x) \frac{t^{i}}{i !} \sum_{j=0}^{\infty}(y)_{j, \lambda} \frac{t^{m}}{m !} \\
& =\sum_{n=0}^{\infty}\left(\sum_{j=0}^{n}\left(\begin{array}{c}
n \\
j
\end{array}\right)(y)_{m, \lambda} \mathfrak{B}_{n-j, \lambda}^{\left(k_{1}, k_{2}, \cdots, k_{r}\right)}(x)\right) \frac{t^{n}}{n !},
\end{aligned}
$$

which implies the desired result (25).

Theorem 8. The following relation

$$
\frac{d}{d x} \mathfrak{B}_{n, \lambda}^{\left(k_{1}, k_{2}, \cdots, k_{r}\right)}(x)={ }_{l=1}^{n}\left(\begin{array}{c}
n \\
l
\end{array}\right) \mathfrak{B}_{n-l, \lambda}^{\left(k_{1}, k_{2}, \cdots, k_{r}\right)}(x)(-\lambda)^{l-1}(l-1) !
$$

is valid for $k_{1}, k_{2}, \cdots, k_{r} \in \mathbb{Z}$ and $n \geq 0$.

Proof. To investigate the derivative property of $\mathfrak{B}_{n, \lambda}^{\left(k_{1}, k_{2}, \cdots, k_{r}\right)}(x)$ that

$$
\begin{aligned}
\sum_{n=0}^{\infty} \frac{d}{d x} \mathfrak{B}_{n, \lambda}^{\left(k_{1}, k_{2}, \cdots, k_{r}\right)}(x) \frac{t^{n}}{n !} & =\frac{r ! \mathrm{Ei}_{k_{1}, k_{2}, \cdots, k_{r} ; \lambda}\left(\log _{\lambda}(1+t)\right)}{\left(e_{\lambda}(t)-1\right)^{r}} \frac{d}{d x} e_{\lambda}^{x}(t) \\
& =\sum_{n=0}^{\infty} \mathfrak{B}_{n, \lambda}^{\left(k_{1}, k_{2}, \cdots, k_{r}\right)}(x) \frac{t^{n}}{n !} \frac{1}{\lambda} \ln (1+\lambda t) \\
& =\left(\sum_{n=0}^{\infty} \mathfrak{B}_{n, \lambda}^{\left(k_{1}, k_{2}, \cdots, k_{r}\right)}(x) \frac{t^{n}}{n !}\right) \sum_{l=1}^{\infty} \frac{(-1)^{l+1}}{l} \lambda^{l-1} t^{l} \\
& =\sum_{n=0}^{\infty} \sum_{l=1}^{\infty} \mathfrak{B}_{n, \lambda}^{\left(k_{1}, k_{2}, \cdots, k_{r}\right)}(x) \frac{(-1)^{l+1}}{l} \lambda^{l-1} \frac{t^{n+l}}{n !},
\end{aligned}
$$

which provides the asserted result (27).

We here give a relation including the degenerate multipoly-Bernoulli polynomials with numbers and the degenerate Stirling numbers of the second kind.

Theorem 9. The following correlation

$$
\mathfrak{B}_{n, \lambda}^{\left(k_{1}, k_{2}, \cdots, k_{r}\right)}(x)=\sum_{m=0}^{n} \sum_{l=0}^{m}\left(\begin{array}{l}
n \\
m
\end{array}\right)(x)_{l} S_{2, \lambda}(m, l) \mathfrak{B}_{n-m, \lambda}^{\left(k_{1}, k_{2}, \cdots, k_{r}\right)},
$$

is valid for $k_{1}, k_{2}, \cdots, k_{r} \in \mathbb{Z}$ and $n \geq 0$.
Proof. By means of Definition 1, we attain that

$$
\begin{aligned}
\sum_{n=0}^{\infty} \mathfrak{B}_{n, \lambda}^{\left(k_{1}, k_{2}, \cdots, k_{r}\right)}(x) \frac{t^{n}}{n !} & =\frac{r ! \mathrm{Ei}_{k_{1}, k_{2}, \cdots, k_{r} ; \lambda}\left(\log _{\lambda}(1+t)\right)}{\left(e_{\lambda}(t)-1\right)^{r}} e_{\lambda}^{x}(t) \\
& =\frac{r ! \mathrm{Ei}_{k_{1}, k_{2}, \cdots, k_{r} ; \lambda}\left(\log _{\lambda}(1+t)\right)}{\left(e_{\lambda}(t)-1\right)^{r}}\left(e_{\lambda}(t)-1+1\right)^{x} \\
& =\frac{r ! \mathrm{Ei}_{k_{1}, k_{2}, \cdots, k_{r} ; \lambda}\left(\log _{\lambda}(1+t)\right)}{\left(e_{\lambda}(t)-1\right)^{r}} \sum_{l=0}^{\infty}\left(\begin{array}{c}
x \\
l
\end{array}\right)\left(e_{\lambda}(t)-1\right)^{l} \\
& =\sum_{n=0}^{\infty} \mathfrak{B}_{n, \lambda}^{\left(k_{1}, k_{2}, \cdots, k_{r}\right)} \frac{t^{n}}{n !} \sum_{l=0}^{\infty}(x)_{l} \sum_{m=l}^{\infty} S_{2, \lambda}(m, l) \frac{t^{m}}{m !} \\
& =\sum_{n=0}^{\infty}\left(\sum_{m=0}^{n} \sum_{l=0}^{m}\left(\begin{array}{c}
n \\
m
\end{array}\right)(x)_{l} S_{2, \lambda}(m, l) \mathfrak{B}_{n-m, \lambda}^{\left(k_{1}, k_{2}, \cdots, k_{r}\right)}\right) \frac{t^{n}}{n !},
\end{aligned}
$$

where the notation $(x)_{l}$ is falling factorial that is defined by $(x)_{0}=1$ and $(x)_{n}=x(x-1) \cdots(x-(n-1))$ for $n \geq 1$, (cf. $[1,2,5-14,21,23,24])$. So, the proof is completed.

Kim [5] introduced the degenerate Whitney numbers are given by

$$
\frac{\left(e_{\lambda}^{m}(t)-1\right)^{k}}{m^{k} k !} e_{\lambda}^{\alpha}(t)=\sum_{n=k}^{\infty} W_{m, \alpha}(n, k \mid \lambda) \frac{t^{n}}{n !},(k \geq 0)
$$

Kim also provided several correlations including the degenerate Stirling numbers of the second kind and the degenerate Whitney numbers (see [5]).

We now give a correlation as follows.

Theorem 10. For $k_{1}, k_{2}, \cdots k_{r} \in \mathbb{Z}$ and $n \geq 0$, we have

$$
\mathfrak{B}_{n, \lambda}^{\left(k_{1}, k_{2}, \cdots, k_{r}\right)}(x u+\alpha)=\sum_{m=0}^{n} \sum_{l=0}^{m}\left(\begin{array}{c}
n \\
m
\end{array}\right) u^{l}(x)_{l} W_{u, \alpha}(m, l \mid \lambda) \mathfrak{B}_{n-m, \lambda}^{\left(k_{1}, k_{2}, \cdots, k_{r}\right)} .
$$

Proof. Using (31) and Definition 1, we acquire that

$$
\begin{aligned}
& \sum_{n=0}^{\infty} \mathfrak{B}_{n, \lambda}^{\left(k_{1}, k_{2}, \cdots, k_{r}\right)}(x u+\alpha) \frac{t^{n}}{n !} \\
& =\frac{r ! \mathrm{Ei}_{k_{1}, k_{2}, \cdots, k_{r} ; \lambda}\left(\log _{\lambda}(1+t)\right)}{\left(e_{\lambda}(t)-1\right)^{r}} e_{\lambda}^{\alpha}(t) e_{\lambda}^{x u}(t) \\
& =\frac{r ! \mathrm{Ei}_{k_{1}, k_{2}, \cdots, k_{r} ; \lambda}\left(\log _{\lambda}(1+t)\right)}{\left(e_{\lambda}(t)-1\right)^{r}} e_{\lambda}^{\alpha}(t)\left(e_{\lambda}^{u}(t)-1+1\right)^{x} \\
& =\frac{r ! \mathrm{Ei}_{k_{1}, k_{2}, \cdots, k_{r} ; \lambda}\left(\log _{\lambda}(1+t)\right)}{\left(e_{\lambda}(t)-1\right)^{r}} e_{\lambda}^{\alpha}(t) \sum_{l=0}^{\infty}\left(\begin{array}{c}
x \\
l
\end{array}\right)\left(e_{\lambda}^{u}(t)-1\right)^{l} \\
& =\frac{r ! \mathrm{Ei}_{k_{1}, k_{2}, \cdots, k_{r} ; \lambda}\left(\log _{\lambda}(1+t)\right)}{\left(e_{\lambda}(t)-1\right)^{r}} \sum_{l=0}^{\infty} u^{l}(x)_{l} \frac{\left(e_{\lambda}^{u}(t)-1\right)^{l}}{l ! u^{l}} e_{\lambda}^{\alpha}(t)
\end{aligned}
$$




$$
\begin{aligned}
& =\frac{r ! \mathrm{Ei}_{k_{1}, k_{2}, \cdots, k_{r} ; \lambda}\left(\log _{\lambda}(1+t)\right)}{\left(e_{\lambda}(t)-1\right)^{r}} \sum_{l=0}^{\infty} u^{l}(x)_{l} \frac{\left(e_{\lambda}^{u}(t)-1\right)^{l}}{l ! u^{l}} e_{\lambda}^{\alpha}(t) \\
& =\sum_{n=0}^{\infty} \mathfrak{B}_{n, \lambda}^{\left(k_{1}, k_{2}, \cdots, k_{r}\right)} \frac{t^{n}}{n !} \sum_{n=0}^{\infty} \sum_{l=0}^{n} u^{l}(x)_{l} W_{u, \alpha}(n, l \mid \lambda) \frac{t^{n}}{n !} \\
& =\sum_{n=0}^{\infty}\left(\sum_{m=0}^{n} \sum_{l=0}^{m}\left(\begin{array}{c}
n \\
m
\end{array}\right) u^{l}(x)_{l} W_{u, \alpha}(m, l \mid \lambda) \mathfrak{B}_{n-m, \lambda}^{\left(k_{1}, k_{2}, \cdots, k_{r}\right)}\right) \frac{t^{n}}{n !}
\end{aligned}
$$

which implies the asserted result (32).

\section{Degenerate Multi-Poly-Bernoulli Polynomials of Complex Variable}

In [25], Kim et al. defined the degenerate $\operatorname{sine} \sin _{\lambda} t$ and cosine $\cos _{\lambda} t$ functions by

$$
\sin _{\lambda}^{(x)}(t)=\frac{e_{\lambda}^{i x}(t)-e_{\lambda}^{-i x}(t)}{2 i} \text { and } \cos _{\lambda}^{(x)}(t)=\frac{e_{\lambda}^{i x}(t)+e_{\lambda}^{-i x}(t)}{2},
$$

where $i=\sqrt{-1}$. Note that $\lim _{\lambda \rightarrow 0} \sin _{\lambda}^{(x)}(t)=\sin x t$ and $\lim _{\lambda \rightarrow 0} \cos _{\lambda}^{(x)}(t)=\cos x t$. From (34), it is readily that

$$
e_{\lambda}^{i x}(t)=\cos _{\lambda}^{(x)}(t)+i \sin _{\lambda}^{(x)}(t)
$$

By these functions in (34), the degenerate sinepolynomials $S_{k, \lambda}(x, y)$ and degenerate cosine-polynomials $C_{k, \lambda}(x, y)$ are introduced (cf. [25]) by

$$
\begin{gathered}
\sum_{n=0}^{\infty} S_{k, \lambda}(x, y) \frac{t^{n}}{n !}=e_{\lambda}^{x}(t) \sin _{\lambda}^{(y)}(t), \\
\sum_{n=0}^{\infty} C_{k, \lambda}(x, y) \frac{t^{n}}{n !}=e_{\lambda}^{x}(t) \cos _{\lambda}^{(y)}(t) .
\end{gathered}
$$

Several properties of these polynomials in (36) and (37) are studied and investigated in [25]. Also, by means of these functions, Kim et al. [25] introduced the degenerate Euler and Bernoulli polynomials of complex variable and investigate some of their properties. Motivated and inspired by these considerations above, we define type 2 degenerate multi-poly-Bernoulli polynomials of complex variable as follows.

Definition 11. Let $k_{1}, k_{2}, \cdots, k_{r} \in \mathbb{Z}$. We define a new form of the degenerate multi-poly-Bernoulli polynomials of complex variable by the following generating function:

$\frac{r ! \mathrm{Ei}_{k_{1}, k_{2}, \cdots, k_{r}, \lambda}\left(\log _{\lambda}(1+t)\right)}{\left(\mathrm{e}_{\lambda}(t)-1\right)^{r}} e_{\lambda}^{x+i y}(t)=\sum_{n=0}^{\infty} \mathrm{B}_{n, \lambda}^{\left(k_{1}, k_{2}, \cdots, k_{r}\right)}(x+i y) \frac{t^{n}}{n !}$.
By (34) and (38), we observe that

$$
\begin{gathered}
\sum_{n=0}^{\infty} \frac{\left(\mathrm{B}_{n, \lambda}^{\left(k_{1}, k_{2}, \cdots, k_{r}\right)}(x+i y)-\mathrm{B}_{n, \lambda}^{\left(k_{1}, k_{2}, \cdots, k_{r}\right)}(x-i y)\right)}{2 i} \frac{t^{n}}{n !} \\
=\frac{r ! \mathrm{Ei}_{k_{1}, k_{2}, \cdots, k_{r} ; \lambda}\left(\log _{\lambda}(1+t)\right)}{\left(e_{\lambda}(t)-1\right)^{r}} e_{\lambda}^{x}(t) \sin _{\lambda}^{(y)}(t),
\end{gathered}
$$

and

$$
\begin{gathered}
\sum_{n=0}^{\infty} \frac{\left(\mathrm{B}_{n, \lambda}^{\left(k_{1}, k_{2}, \cdots, k_{r}\right)}(x+i y)+\mathrm{B}_{n, \lambda}^{\left(k_{1}, k_{2}, \cdots, k_{r}\right)}(x-i y)\right)}{2} \frac{t^{n}}{n !} \\
=\frac{r ! \mathrm{Ei}_{k_{1}, k_{2}, \cdots, k_{r} ; \lambda}\left(\log _{\lambda}(1+t)\right)}{\left(e_{\lambda}(t)-1\right)^{r}} e_{\lambda}^{x}(t) \cos _{\lambda}^{(y)}(t) .
\end{gathered}
$$

In view of (39) and (40), we consider the degenerate multi-poly-sine-Bernoulli polynomials $\mathrm{B}_{n, \lambda}^{\left(k_{1}, k_{2}, \cdots, k_{r} ; S\right)}(x, y)$ with two parameters and the degenerate multi-poly-cosineBernoulli polynomials $\mathrm{B}_{n, \lambda}^{\left(k_{1}, k_{2}, \cdots, k_{r} ; C\right)}(x, y)$ with two parameters as follows:

$\sum_{n=0}^{\infty} \mathrm{B}_{n, \lambda}^{\left(k_{1}, \mathrm{k}_{2}, \cdots, k_{r} ; S\right)}(x, y) \frac{t^{n}}{n !}=\frac{r ! \mathrm{Ei}_{k_{1}, k_{2}, \cdots, k_{r} ; \lambda}\left(\log _{\lambda}(1+t)\right)}{\left(e_{\lambda}(t)-1\right)^{r}} e_{\lambda}^{x}(t) \sin _{\lambda}^{(y)}(t)$,

$\sum_{n=0}^{\infty} \mathrm{B}_{n, \lambda}^{\left(k_{1}, k_{2}, \cdots, k_{r} ; C\right)}(x, y) \frac{t^{n}}{n !}=\frac{r ! \mathrm{Ei}_{k_{1}, k_{2}, \cdots, k_{r} ; \lambda}\left(\log _{\lambda}(1+t)\right)}{\left(e_{\lambda}(t)-1\right)^{r}} e_{\lambda}^{x}(t) \cos _{\lambda}^{(y)}(t)$.

Note that

$$
\begin{array}{r}
\lim _{\lambda \rightarrow 0} \mathrm{~B}_{n, \lambda}^{\left(k_{1}, k_{2}, \cdots, k_{r} ; S\right)}(x, y):=\mathrm{B}_{n}^{\left(k_{1}, k_{2}, \cdots, k_{r} ; S\right)}(x, y) \text { and } \\
\lim _{\lambda \rightarrow 0} \mathrm{~B}_{n, \lambda}^{\left(k_{1}, k_{2}, \cdots, k_{r} ; C\right)}(x, y):=\mathrm{B}_{n}^{\left(k_{1}, k_{2}, \cdots, k_{r} ; C\right)}(x, y),
\end{array}
$$

which are multi-poly-sine-Bernoulli polynomials $\mathrm{B}_{n}^{\left(k_{1}, k_{2}, \cdots, k_{r} ; S\right)}(x, y)$ and multi-poly-cosine-Bernoulli polynomials $\mathrm{B}_{n}^{\left(k_{1}, k_{2}, \cdots, k_{r} ; C\right)}(x, y)$ with two parameters.

By (39)-(42), we see that

$$
\begin{aligned}
& \mathrm{B}_{n, \lambda}^{\left(k_{1}, k_{2}, \cdots, k_{r} ; S\right)}(x, y)=\frac{\left(\mathrm{B}_{n, \lambda}^{\left(k_{1}, k_{2}, \cdots, k_{r}\right)}(x+i y)-\mathrm{B}_{n, \lambda}^{\left(k_{1}, k_{2}, \cdots, k_{r}\right)}(x-i y)\right)}{2 i}, \\
& \mathrm{~B}_{n, \lambda}^{\left(k_{1}, k_{2}, \cdots, k_{r} ; C\right)}(x, y)=\frac{\left(\mathrm{B}_{n, \lambda}^{\left(k_{1}, k_{2}, \cdots, k_{r}\right)}(x+i y)+\mathrm{B}_{n, \lambda}^{\left(k_{1}, k_{2}, \cdots, k_{r}\right)}(x-i y)\right)}{2} .
\end{aligned}
$$

We now give the two summation formulae by the following theorem. 
Theorem 12. For $k_{1}, k_{2}, \cdots, k_{r} \in \mathbb{Z}$ and $n \geq 0$, we have

$$
\begin{aligned}
& B_{n, \lambda}^{\left(k_{1}, k_{2}, \cdots, k_{r}\right)}(x+i y)=\sum_{m=0}^{n}\left(\begin{array}{c}
n \\
m
\end{array}\right) B_{n-m, \lambda}^{\left(k_{1}, k_{2}, \cdots k_{r}\right)}(x)(i y)_{m, \lambda}, \\
& B_{n, \lambda}^{\left(k_{1}, k_{2}, \cdots, k_{r}\right)}(x+i y)=\sum_{m=0}^{n}\left(\begin{array}{c}
n \\
m
\end{array}\right) B_{n-m, \lambda}^{\left(k_{1}, k_{2}, \cdots k_{r}\right)}(x+i y)_{m, \lambda} .
\end{aligned}
$$

Proof. The proofs of this theorem can be done by using the same proof methods used in Theorems 5 and 7. So, we omit the proofs.

We here provide the two derivative formulae by the following theorem.

Theorem 13. For $k_{1}, k_{2}, \cdots, k_{r} \in \mathbb{Z}$ and $n \geq 0$, we have

$$
\begin{gathered}
\frac{d}{d t} B_{n, \lambda}^{\left(k_{1}, k_{2}, \cdots, k_{r}\right)}(x+i y)=n B_{n-1, \lambda}^{\left(k_{1}, k_{2}, \cdots, k_{r}\right)}(x+i y) \\
\frac{d}{d x} \mathfrak{B}_{n, \lambda}^{\left(k_{1}, k_{2}, \cdots, k_{r}\right)}(x+i y)={ }_{l=1}^{n}\left(\begin{array}{c}
n \\
l
\end{array}\right) \mathfrak{B}_{n-l, \lambda}^{\left(k_{1}, k_{2}, \cdots, k_{r}\right)}(x+i y)(-\lambda)^{l-1}(l-1) !
\end{gathered}
$$

Proof. The proofs of this theorem can be done by using the same proof methods used in Theorem 8. So, we omit the proofs.

We give the following theorem.

Theorem 14. For $k_{1}, k_{2}, \cdots, k_{r} \in \mathbb{Z}$ and $n \geq 0$, we have

$$
\begin{aligned}
& B_{n, \lambda}^{\left(k_{1}, k_{2}, \cdots, k_{r} ; S\right)}(x, y)={ }_{l=0}^{n}\left(\begin{array}{c}
n \\
l
\end{array}\right) B_{n-l, \lambda}^{\left(k_{1}, k_{2}, \cdots, k_{r}\right)} S_{l, \lambda}(x, y), \\
& B_{n, \lambda}^{\left(k_{1}, k_{2}, \cdots, k_{r} ; C\right)}(x, y)={ }_{l=0}^{n}\left(\begin{array}{c}
n \\
l
\end{array}\right) B_{n-l, \lambda}^{\left(k_{1}, k_{2}, \cdots, k_{r}\right)} C_{l, \lambda}(x, y) .
\end{aligned}
$$

Proof. From (36), (37), and (38), we get

$$
\begin{aligned}
& \sum_{n=0}^{\infty} \mathrm{B}_{n, \lambda}^{\left(k_{1}, k_{2}, \cdots, k_{r} ; S\right)}(x, y) \frac{t^{n}}{n !} \\
& =\frac{r ! \mathrm{Ei}_{k_{1}, k_{2}, \cdots, k_{r} ; \lambda}\left(\log _{\lambda}(1+t)\right)}{\left(e_{\lambda}(t)-1\right)^{r}} e_{\lambda}^{x}(t) \sin _{\lambda}^{(y)}(t) \\
& =\sum_{n=0}^{\infty} \mathrm{B}_{n, \lambda}^{\left(k_{1}, k_{2}, \cdots, k_{r}\right)} \frac{t^{n}}{n !} \sum_{n=0}^{\infty} S_{n, \lambda}(x, y) \frac{t^{n}}{n !} \\
& ={ }_{n=0}^{\infty}\left(\begin{array}{l}
n \\
l=0
\end{array}\left(\begin{array}{l}
n \\
l
\end{array}\right) \mathrm{B}_{n-l, \lambda}^{\left(k_{1}, k_{2}, \cdots, k_{r}\right)} S_{l, \lambda}(x, y)\right) \frac{t^{n}}{n !},
\end{aligned}
$$

$$
\begin{aligned}
& \sum_{n=0}^{\infty} \mathrm{B}_{n, \lambda}^{\left(k_{1}, k_{2}, \cdots, k_{r} ; C\right)}(x, y) \frac{t^{n}}{n !} \\
& =\frac{r ! \mathrm{Ei}_{k_{1}, k_{2}, \cdots, k_{r} ; \lambda}\left(\log _{\lambda}(1+t)\right)}{\left(e_{\lambda}(t)-1\right)^{r}} e_{\lambda}^{x}(t) \cos _{\lambda}^{(y)}(t) \\
& =\sum_{n=0}^{\infty} \mathrm{B}_{n, \lambda}^{\left(k_{1}, k_{2}, \cdots, k_{r}\right)} \frac{t^{n}}{n !} \sum_{n=0}^{\infty} C_{n, \lambda}(x, y) \frac{t^{n}}{n !} \\
& ={ }_{n=0}^{\infty}\left(\begin{array}{l}
n \\
l=0
\end{array}\left(\begin{array}{l}
n \\
l
\end{array}\right) \mathrm{B}_{n-l, \lambda}^{\left(k_{1}, k_{2}, \cdots, k_{r}\right)} C_{l, \lambda}(x, y)\right) \frac{t^{n}}{n !},
\end{aligned}
$$

which complete the proof of the theorem.

We note that $(c f .[25])$

$$
\begin{gathered}
\sin _{\lambda}^{(y)}(t)={ }_{n=1 k=0}^{\infty} \lambda^{\left[\frac{n-1}{2}\right]} \lambda^{n-2 k-1}(-1)^{k} y^{2 k+1} S_{1, \lambda}(n, 2 k+1) \frac{t^{n}}{n !}, \\
\cos _{\lambda}^{(y)}(t)={ }_{n=0 k=0}^{\infty}\left[\frac{n}{2}\right] \\
\lambda^{n-2 k}(-1)^{k} y^{2 k} S_{1, \lambda}(n, 2 k) \frac{t^{n}}{n !} .
\end{gathered}
$$

We give the following theorem.

Theorem 15. For $k_{1}, k_{2}, \cdots, k_{r} \in \mathbb{Z}$ and $n \geq 0$, we have

$$
\begin{aligned}
B_{n, \lambda}^{\left(k_{1}, k_{2}, \cdots, k_{r} ; S\right)}(x, y)= & { }_{l=1 k=0}^{n\left[\frac{l-1}{2}\right]}\left(\begin{array}{c}
n \\
l
\end{array}\right) B_{n-l, \lambda}^{\left(k_{1}, k_{2}, \cdots, k_{r}\right)} \\
& \cdot(x) \lambda^{l-2 k-1}(-1)^{k} y^{2 k+1} S_{1, \lambda}(l, 2 k+1),
\end{aligned}
$$

$$
\begin{aligned}
B_{n, \lambda}^{\left(k_{1}, k_{2}, \cdots, k_{r} ; C\right)}(x, y)= & { }_{l=1 k=0}^{n\left[\frac{l}{2}\right]}\left(\begin{array}{c}
n \\
l
\end{array}\right) B_{n-l, \lambda}^{\left(k_{1}, k_{2}, \cdots, k_{r}\right)} \\
& \cdot(x) \lambda^{l-2 k-1}(-1)^{k} y^{2 k+1} S_{1, \lambda}(l, 2 k+1),
\end{aligned}
$$

where the notation [.] is Gauss' notation and represents the maximum integer which does not exceed a number in the square bracket.

Proof. By (41)-(51), we observe that

$$
\begin{aligned}
& \sum_{n=0}^{\infty} \mathrm{B}_{n, \lambda}^{\left(k_{1}, k_{2}, \cdots, k_{r} ; S\right)}(x, y) \frac{t^{n}}{n !} \\
& \quad=\sum_{n=0}^{\infty} \mathrm{B}_{n, \lambda}^{\left(k_{1}, k_{2}, \cdots, k_{r}\right)}(x) \frac{t^{n \infty}}{n !}{ }_{n=1}^{\left.n \frac{n-1}{2}\right]} \lambda^{n-2 k-1}(-1)^{k} y^{2 k+1} S_{1, \lambda}(n, 2 k+1) \frac{t^{n}}{n !} \\
& \quad={ }_{n=0}^{\infty}\left(\begin{array}{c}
n \\
l=1
\end{array}\left(\begin{array}{c}
n \\
l
\end{array}\right) \mathrm{B}_{n-l, \lambda}^{\left(k_{1}, k_{2}, \cdots, k_{r}\right)}(x)_{k=0}^{\left[\frac{l-1}{2}\right]} \lambda^{l-2 k-1}(-1)^{k} y^{2 k+1} S_{1, \lambda}(l, 2 k+1)\right) \frac{t^{n}}{n !},
\end{aligned}
$$




$$
\begin{aligned}
& \sum_{n=0}^{\infty} \mathrm{B}_{n, \lambda}^{\left(k_{1}, k_{2}, \cdots, k_{r}, C\right)}(x, y) \frac{t^{n}}{n !} \\
& =\frac{r ! \mathrm{Ei}_{k_{1}, k_{2}, \cdots, k_{r} ; \lambda}\left(\log _{\lambda}(1+t)\right)}{\left(e_{\lambda}(t)-1\right)^{r}} e_{\lambda}^{x}(t) \cos _{\lambda}^{(y)}(t)
\end{aligned}
$$

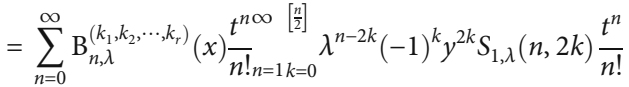

$$
\begin{aligned}
& ={ }_{n=0}^{\infty}\left(\begin{array}{l}
n \\
l=1
\end{array}\left(\begin{array}{l}
n \\
l
\end{array}\right) \mathrm{B}_{n-l, \lambda}^{\left(k_{1}, k_{2}, \cdots, k_{r}\right)}(x)_{k=0}^{\left[\frac{1}{2}\right]} \lambda^{l-2 k}(-1)^{k} y^{2 k} S_{1, \lambda}(l, 2 k)\right) \frac{t^{n}}{n !} .
\end{aligned}
$$

So, the proof is completed.

We give the following proposition.

Proposition 16. The following relations

$$
\begin{aligned}
& B_{n, \lambda}^{\left(k_{1}, k_{2}, \cdots, k_{r} ; S\right)}(x+u, y)={ }_{l=0}^{n}\left(\begin{array}{l}
n \\
l
\end{array}\right) B_{n-l, \lambda}^{\left(k_{1}, k_{2}, \cdots, k_{r} ; S\right)}(x, y)(u)_{l, \lambda}, \\
& B_{n, \lambda}^{\left(k_{1}, k_{2}, \cdots, k_{r} ; C\right)}(x+u, y)={ }_{l=0}^{n}\left(\begin{array}{l}
n \\
l
\end{array}\right) B_{n-l, \lambda}^{\left(k_{1}, k_{2}, \cdots, k_{r} ; C\right)}(x, y)(u)_{l, \lambda} .
\end{aligned}
$$

hold for $k_{1}, k_{2}, \cdots, k_{r} \in \mathbb{Z}, u \in \mathbb{C}$ and $n \geq 0$.

Proof. The proofs of this proposition can be done by utilizing the same proof methods used in Theorem 7. So, we omit the proofs.

Upon setting $x=0$ in (41) and (42), we consider the degenerate multi-poly-sine-Bernoulli polynomials $\mathrm{B}_{n, \lambda}^{\left(k_{1}, k_{2}, \cdots, k_{r} ; S\right)}(y)$ and the degenerate multi-poly-cosine-Bernoulli polynomials $\mathrm{B}_{n, \lambda}^{\left(k_{1}, k_{2}, \cdots, k_{r} ; C\right)}(y)$ as follows

$$
\sum_{n=0}^{\infty} \mathrm{B}_{n, \lambda}^{\left(k_{1}, k_{2}, \cdots, k_{r}, S\right)}(y) \frac{t^{n}}{n !}=\frac{r ! \mathrm{Ei}_{k_{1}, k_{2}, \cdots, k_{r}, \lambda}\left(\log _{\lambda}(1+t)\right)}{\left(e_{\lambda}(t)-1\right)^{r}} \sin _{\lambda}^{(y)}(t),
$$

$\sum_{n=0}^{\infty} \mathrm{B}_{n, \lambda}^{\left(k_{1}, k_{2}, \cdots, k_{r} ; C\right)}(y) \frac{t^{n}}{n !}=\frac{r ! \mathrm{Ei}_{k_{1}, k_{2}, \cdots, k_{r} ; \lambda}\left(\log _{\lambda}(1+t)\right)}{\left(e_{\lambda}(t)-1\right)^{r}} \cos _{\lambda}^{(y)}(t)$.

We now provide the following theorem.

Theorem 17. For $k_{1}, k_{2}, \cdots, k_{r} \in \mathbb{Z}$ and $n>0$, we have

$$
\begin{aligned}
\mathfrak{B}_{n, \lambda}^{\left(k_{1}, k_{2}, \cdots, k_{r} ; C\right)}(x, y)= & \sum_{m=0}^{n} \sum_{l=0}^{m}\left(\begin{array}{c}
n \\
m
\end{array}\right)(x)_{l} S_{2, \lambda}(m, l) \mathfrak{B}_{n-m, \lambda}^{\left(k_{1}, k_{2}, \cdots, k_{r} ; C\right)} \\
& \cdot(y) \text { with } \boldsymbol{B}_{n, \lambda}^{\left(k_{1}, k_{2}, \cdots, k_{r} ; C\right)}(x, y)=0 .
\end{aligned}
$$

Proof. The proofs of this theorem can be done by utilizing the same proof methods in Theorem 9.

Let $\alpha$ be any fixed real (or complex) number. The Bernoulli polynomials of order $\alpha$ is defined by (cf. [25])

$$
\left(\frac{t}{e^{t}-1}\right)^{\alpha} e^{x t}=\sum_{n=0}^{\infty} B_{n}^{(\alpha)}(x) \frac{t^{n}}{n !}, \quad(|t|<2 \pi) .
$$

When $x=0$, the Bernoulli polynomials of order $\alpha$ reduce to the Bernoulli numbers of order $\alpha$, denoted by $B_{n}^{(\alpha)}$.We give the following relation.

Theorem 18. For $k_{1}, k_{2}, \cdots, k_{r} \in \mathbb{Z}$ and $n \geq 0$, we have

$$
\begin{aligned}
B_{n, \lambda}^{\left(k_{1}, k_{2}, \cdots, k_{r} ; S\right)} & (1, y)-B_{n, \lambda}^{\left(k_{1}, k_{2}, \cdots, k_{r} ; S\right)}(y) \\
& =n \sum_{l=0}^{n-1}\left(\begin{array}{c}
n-1 \\
l
\end{array}\right) B_{n-1-l, \lambda}^{\left(k_{1}, k_{2}, \cdots, k_{r} ; S\right)}(y) B_{l}^{(-1)}, \\
B_{n, \lambda}^{\left(k_{1}, k_{2}, \cdots, k_{r} ; C\right)}(1, y)-B_{n, \lambda}^{\left(k_{1}, k_{2}, \cdots, k_{r} ; C\right)}(y) & \\
= & n \sum_{l=0}^{n-1}\left(\begin{array}{c}
n-1 \\
l
\end{array}\right) B_{n-1-l, \lambda}^{\left(k_{1}, k_{2}, \cdots, k_{r} ; C\right)}(y) B_{l}^{(-1)} .
\end{aligned}
$$

Proof. By (55) and (56), we acquire

$$
\begin{aligned}
& \sum_{n=0}^{\infty} \mathrm{B}_{n, \lambda}^{\left(k_{1}, k_{2}, \cdots, k_{r} ; S\right)}(1, y) \frac{t^{n}}{n !}-\sum_{n=0}^{\infty} \mathrm{B}_{n, \lambda}^{\left(k_{1}, k_{2}, \cdots, k_{r} ; S\right)}(y) \frac{t^{n}}{n !} \\
& \quad=\frac{r ! \mathrm{Ei}_{k_{1}, k_{2}, \cdots, k_{r} ; \lambda}\left(\log _{\lambda}(1+t)\right)}{\left(e_{\lambda}(t)-1\right)^{r}} \sin _{\lambda}^{(y)}(t)\left(e_{\lambda}(t)-1\right) \\
& \quad=\sum_{n=0}^{\infty} \mathrm{B}_{n, \lambda}^{\left(k_{1}, k_{2}, \cdots, k_{r} ; S\right)}(y) \frac{t^{n+1}}{n !} \sum_{n=0}^{\infty} B_{n}^{(-1)} \frac{t^{n}}{n !} \\
& =\sum_{n=0}^{\infty} \sum_{l=0}^{n}\left(\begin{array}{l}
n \\
l
\end{array}\right) \mathrm{B}_{n-l, \lambda}^{\left(k_{1}, k_{2}, \cdots, k_{r} ; S\right)}(y) B_{l}^{(-1)} \frac{t^{n+1}}{n !} .
\end{aligned}
$$

Thus, (59) is proved. We prove (60) in the same way.

Here is a special case of Theorem 18.

Corollary 19. For $k_{1}, k_{2}, \cdots, k_{r} \in \mathbb{Z}$ and $n \geq 0$, we have $B_{n, \lambda}^{\left(k_{1}, k_{2}, \cdots, k_{r}\right)}(1)-B_{n, \lambda}^{\left(k_{1}, k_{2}, \cdots, k_{r}\right)}=n \sum_{l=0}^{n-1}\left(\begin{array}{c}n-1 \\ l\end{array}\right) B_{n-1-l, \lambda}^{\left(k_{1}, k_{2}, \cdots, k_{r}\right)} B_{l}^{(-1)}$,

which is a relation including the degenerate multi-polyBernoulli polynomials.

\section{Conclusions}

In this paper, we defined the degenerate multi-poly-Bernoulli polynomials by employing the degenerate multiple 
polyexponential functions. We have established some identities and relations between degenerate Whitney numbers and degenerate Stirling numbers of the first kind. Also, we have established addition formulas and derivative formulas of degenerate multi-poly-Bernoulli polynomials. In the last section, we have defined degenerate multi-poly-Bernoulli polynomials of complex variables and then we have derived several properties and relations.

\section{Data Availability}

No data were used to support this study.

\section{Conflicts of Interest}

The authors declare that there are no conflicts of interest.

\section{Acknowledgments}

This work was supported by the Taif University Researchers Supporting Project (TURSP-2020/246), Taif University, Taif, Saudi Arabia.

\section{References}

[1] L. Carlitz, "Degenerate Stirling," Bernoulli and Eulerian numbers. Utilitas Math., vol. 15, pp. 51-88, 1979.

[2] L. Carlitz, "A degenerate Staudt-Clausen theorem," Archiv der Mathematik, vol. 7, no. 1, pp. 28-33, 1956.

[3] H. Haroon and W. A. Khan, "Degenerate Bernoulli numbers and polynomials associated with degenerate Hermite polynomials," Communication of the Korean Mathematical Society, vol. 33, no. 2, pp. 651-669, 2018.

[4] W. A. Khan, M. Acikgoz, and U. Duran, "Note on the type 2 degenerate multi-poly-Euler polynomials," Symmetry, vol. 12, no. 1691 , pp. 1-10, 2020.

[5] T. Kim, "A note on degenerate Stirling polynomials of the second kind," Proc. Jangjeon Math. Soc., vol. 20, no. 3, pp. 319-331, 2017.

[6] T. Kim, L.-C. Jang, D. S. Kim, and H.-Y. Kim, "Some identities on type 2 degenerate Bernoulli polynomials of the second kind," Symmetry, vol. 12, no. 4, p. 510, 2020.

[7] T. Kim and D. S. Kim, "Degenerate polyexponential functions and degenerate Bell polynomials," Journal of Mathematical Analysis and Applications, vol. 487, no. 2, p. 124017, 2020.

[8] D. S. Kim and T. Kim, "A note on a new type of degenerate Bernoulli numbers," Russian Journal of Mathematical Physics, vol. 27, no. 2, pp. 227-235, 2020.

[9] T. Kim, D. S. Kim, H. Y. Kim, and J. Kwon, "Degenerate Stirling polynomials of the second kind and some applications," Symmetry, vol. 11, no. 8, p. 1046, 2019.

[10] T. Kim and D. S. Kim, "Degenerate Laplace transform and degenerate gamma function," Russian Journal of Mathematical Physics, vol. 24, no. 2, pp. 241-248, 2017.

[11] T. Kim, W. A. Khan, S. K. Sharma, and M. Ghayasuddin, "A note on parametric kinds of the degenerate poly-Bernoulli and poly-Genocchi polynomials," Symmetry, vol. 12, no. 4, p. $614,2020$.

[12] T. Kim, D. S. Kim, J. Kwon, and H. Lee, "Degenerate polyexponential functions and type 2 degenerate poly-Bernoulli num- bers and polynomials," Advances in Difference Equations, vol. 2020, no. 1, 2020.

[13] T. Kim, D. S. Kim, J. Kwon, and H. Y. Kim, “A note on degenerate Genocchi and poly-Genocchi numbers and polynomials," Journal of Inequalities and Applications, vol. 2020, no. $1,2020$.

[14] T. Kim and D. S. Kim, "A note on degenerate multi-polyBernoulli numbers and polynomials," 2020, http://arxiv.org/ abs/2005.07319.

[15] T. Kim, D. S. Kim, H.-Y. Kim, and J. Kwon, "A note on degenerate multi-poly-Genocchi polynomials," Advanced Studies in Contemporary Mathematics, vol. 30, no. 3, pp. 447-454, 2020.

[16] M. M. Jamei, M. R. Beyki, and W. Koepf, "An extension of the Euler-Maclaurin quadrature formula using a parametric type of Bernoulli polynomials," Bulletin des Sciences Mathematiques, vol. 156, p. 102798, 2019.

[17] W. A. Khan, "Degenerate Hermite-Bernoulli numbers and polynomials of the second kind," Prespacetime Journal, vol. 7, no. 9, pp. 1297-1305, 2016.

[18] W. A. Khan, M. Ghayasuddin, and M. Shadab, "Multiple polyBernoulli polynomials of the second kind associated with Hermite polynomials," Fasciculi Mathematici, vol. 58, no. 1, pp. 97-112, 2017.

[19] W. A. Khan, M. Ghayasuddin, N. Khan, and J. Choi, "The 3-variable Hermite poly-Bernoulli polynomials of the second kind," Nonlinear Functional Analysis and Applications, vol. 22, no. 5, pp. 1013-1027, 2017.

[20] D. S. Kim and T. Kim, "Some identities for Bernoulli numbers of the second kind arising from a non-linear differential equation," Bulletin of the Korean Mathematical Society, vol. 52, no. 6, pp. 2001-2010, 2015.

[21] D. S. Kim and T. Kim, "A note on polyexponential and unipoly functions," Russian Journal of Mathematical Physics, vol. 26, no. 1, pp. 40-49, 2019.

[22] T. Kim, H.-I. Kwon, S.-H. Lee, and J. I. Seo, "A note on polyBernoulli numbers and polynomials of the second kind," Advances in Difference Equations, vol. 2014, no. 1, 2014.

[23] D. V. Dolgy and L. C. Jang, "Some identities on the polyGenocchi polynomials and numbers," Symmetry, vol. 12, no. 6, p. 1007, 2020

[24] U. Duran, M. Acikgoz, and S. Araci, "Construction of the type 2 poly-Frbenius-Genocchi polynomials with their certain applications," Advances in Differences Equations, vol. 2020, no. 432, pp. 1-14, 2020.

[25] D. S. Kim, T. Kim, and H. Lee, "A note on degenerate Euler and Bernoulli polynomials of complex variable," Symmetry, vol. 11, no. 9, p. 1168, 2019.

[26] F. Qi, D. S. Kim, and T. Kim, "Multiple poly-Bernoulli polynomials of the second kind," Advanced Studies in Contemporary Mathematics, vol. 25, pp. 1-7, 2015.

[27] S. Roman, The Umbral Calculus, Academic Press, Inc., New York, NY, USA, 1984.

[28] G. Muhiuddin, W. A. Khan, U. Duran, and D. Al-Kadi, “A new class of higher-order hypergeometric bernoulli polynomials associated with lagrange-hermite polynomials," Symmetry, vol. 13, no. 4, p. 648, 2021.

[29] G. Muhiuddin, W. A. Khan, and U. Duran, "Two-variable yype 2 poly-fubini polynomials," Mathematics, vol. 9, no. 3, p. 281, 2021. 